\title{
Time-Resolved Studies of Gallium Nitride Doped with Gadolinium
}

\author{
B. WiteK ${ }^{a}$, A. Wysmolek ${ }^{a, *}$, M. KAmińskA ${ }^{a}$, \\ A. Twardowski ${ }^{a}$, M. Potemski $^{b}$, M. BoćKowski $^{c}$ \\ AND I. GRZEGORY ${ }^{c}$ \\ ${ }^{a}$ Institute of Experimental Physics, University of Warsaw \\ Hoża 69, 00-681 Warsaw, Poland \\ ${ }^{b}$ Grenoble High Magnetic Fields Laboratory \\ BP 166X, F-38042 Grenoble Cedex 9, France \\ ${ }^{c}$ Institute of High Pressure Physics, Polish Academy of Sciences, Warsaw, Poland \\ Time-resolved photoluminescence experiments on high quality bulk GaN \\ doped with Gd are presented. It was found that the decay time of Gd-related \\ transitions observed for $4.2 \mathrm{~K}$ around $1.78 \mathrm{eV}$ is of about $3 \mathrm{~ms}$. Such a long \\ decay time strongly supports the identification of this emission band as due \\ to transitions between $\mathrm{Gd}^{3+}\left(4 f^{7}\right)$ levels. The decay time measured for Gd- \\ -related transitions observed in the UV spectral range, close to the GaN \\ band-gap, was found to be much faster than $1 \mu \mathrm{s}$. This suggests that these \\ emission lines could hardly be correlated with internal transitions within \\ $\mathrm{Gd}^{3+}\left(4 f^{7}\right)$. Possible origin of the Gd-related UV luminescence is discussed. \\ PACS numbers: 78.55.Cr, 75.50.Pp, 71.55.-i, 71.35.Ji
}

\section{Introduction}

Over the last few years, the above room temperature ferromagnetism in MBE-grown GaN layers weakly doped with Gd has been reported by different researchers. The estimated average value of the magnetic moment per Gd ion has been deduced to be as high as 500 times larger as that of the Gd atomic moment [1]. It has been argued that the observed enhancement of the average magnetic moment resulted from strong contribution of GaN host lattice to macroscopic magnetization of the whole material. The origin of such behavior remains puzzling and more studies of GaN:Gd are required to clarify this issue.

\footnotetext{
*corresponding author; e-mail: Andrzej.Wysmolek@fuw.edu.pl
} 
Recently, we have shown that $\mathrm{Gd}^{3+}$ ions could be successfully introduced into bulk GaN crystals, grown from the solution of nitrogen in liquid gallium under high $(1.5 \mathrm{GPa})$ pressure $(\mathrm{HP})$ of $\mathrm{N}_{2}$ and at elevated temperatures of about $1500^{\circ} \mathrm{C}[2]$. The presence of $\mathrm{Gd}$ in these crystals was confirmed by electron paramagnetic resonance (EPR) and photoluminescence (PL) studies. In EPR spectrum, sharp absorption lines were detected, which could be attributed to $\mathrm{Gd}^{3+}\left(4 f^{7}\right)$ state [2]. PL studies of Gd-doped GaN crystals revealed new narrow transitions, close to the GaN band gap (3.1-3.6 eV) and in the range of 1.6-1.8 eV. This latter emission band consists of several lines with a dominant one at $1.78 \mathrm{eV}$ (as narrow as 4-5 meV). Luminescence studies of AlN doped with gadolinium showed that the ${ }^{8} S_{7 / 2}$ ground state and the nearest excited ${ }^{6} P_{J}(J=7 / 2,5 / 2,3 / 2)$ states of $\mathrm{Gd}^{3+}$ are separated by about $3.9 \mathrm{eV}[3]$. Therefore $1.78 \mathrm{eV}$ emission line can be hardly assigned to the ${ }^{8} S_{7 / 2} \rightarrow{ }^{6} P_{J}$ transition. However, the red emission band at energy of about $2 \mathrm{eV}$ was observed for $\mathrm{LiYF}_{4}$ crystals doped with gadolinium and identified as due to the transitions between ${ }^{6} G_{7 / 2}$ and ${ }^{6} P_{J}$ excited states of $\mathrm{Gd}^{3+}[3]$. Thus, by analogy with $\mathrm{LiYF}_{4}$ we have assigned the $1.6-1.8 \mathrm{eV}$ emission band, in our GaN:Gd crystals, to the transitions between ${ }^{6} G_{7 / 2}$ and ${ }^{6} P_{J}$ excited states of $\mathrm{Gd}^{3+}[2]$. This tentative identification needs to be further verified.

The origin of the Gd-related emission lines observed in UV region is highly speculative. The corresponding energy positions seem to be too low to be assigned to the ${ }^{6} P_{7 / 2} \rightarrow{ }^{8} S_{7 / 2}$ transition involving the first excited and the ground state of the $\mathrm{Gd}^{3+}\left(4 f^{7}\right)$ ion, which has already been observed for AlN:Gd [3].

In order to clarify the nature of the observed transitions, time-resolved photoluminescence experiments on high quality bulk GaN doped with Gd were performed.

\section{Samples and experiment}

Strain free GaN bulk crystals of wurtzite structure doped with Gd were grown at High Pressure Research Center as described in Ref. [2]. Time resolved experiments in the UV region were performed using $3.813 \mathrm{eV}$ line of $\mathrm{He}-\mathrm{Cd}$ laser coupled to an acousto-optic modulator providing time resolution better than $1 \mu \mathrm{s}$. The exciting light was focused on the sample using microscope objective, providing spot size of a few microns. Gated photon counting system with photomultiplier was used for signal detection. For the time resolved measurements in the red spectral range pulsed Nd:YLF laser operating at $523 \mathrm{~nm}$ was used. The spectra were collected using intensified CCD detector providing time resolution of about $10 \mathrm{~ns}$.

\section{Results and discussion}

The time evolution of the main luminescence line in the red part of the Gd related emission is presented in Fig. 1. It is clearly seen that overall decay time is in the range of $\mathrm{ms}$. The origin of the additional shoulder observed a few $\mathrm{meV}$ above 


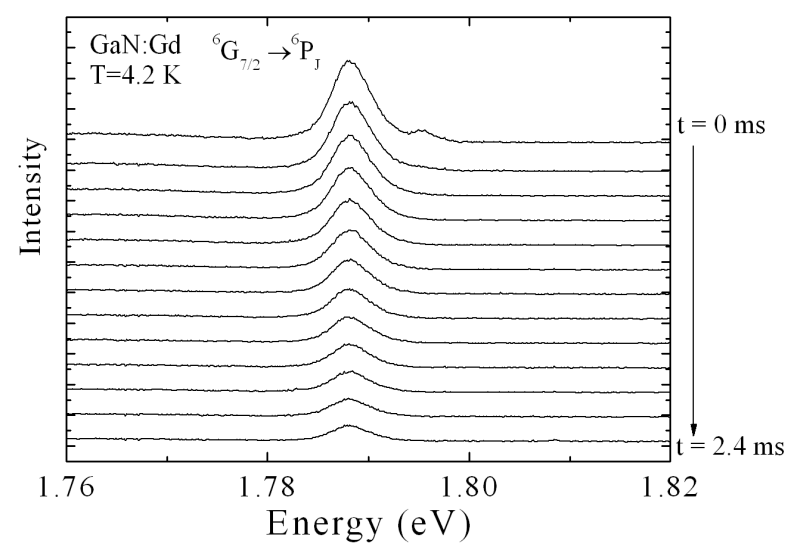

Fig. 1. Time evolution of the main luminescence line in the red band attributed to ${ }^{6} G_{7 / 2} \rightarrow{ }^{6} P_{J}$ transition within $\mathrm{Gd}^{3+}\left(4 f^{7}\right)$ ion.

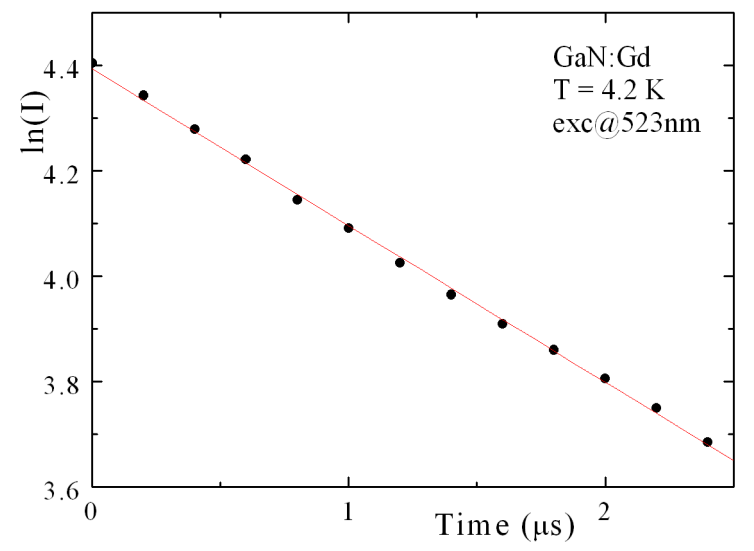

Fig. 2. Time dependence measured for the main line in the red part of the Gd-related emission.

the main transition is not identified yet, and could be related to some parasitic emission in the experimental system.

The logarithmic plot of the intensity of the $1.784 \mathrm{eV}$ line is presented in Fig. 2. The observed time-dependence is linear, which corresponds to the monoexponential decay process. From the fitting to the experimental data the characteristic decay time of $(3.4 \pm 0.1) \mathrm{ms}$ was obtained. Such a long decay time supports the identification of this emission band as due to internal transitions between $\mathrm{Gd}^{3+}\left(4 f^{7}\right)$ levels, for which the decay time in the ms range is observed in the case of many compounds doped with Gd. To firmly assign the $1.78 \mathrm{eV}$ emission to the gadolinium center, the possibility of emission from other rare earth ions, which could be present as impurities in our GaN crystals, should be excluded. According to the available experimental data, the spectral positions of the emis- 
sion lines, as well as intensity patterns observed in $\mathrm{GaN}$ due to $\mathrm{Eu}^{3+}$ [4], $\mathrm{Tb}^{3+}$ [5], $\mathrm{Dy}^{3+}[6], \mathrm{Sm}^{3+}$ [7] ions, which would give emission in the red region, as well as $\operatorname{Er}^{3+}[6,8-10], \mathrm{Tm}^{3+}[11], \mathrm{Nd}^{3+}[12,13]$ and $\operatorname{Pr}^{3+}[14]$ do not coincide with $1.78 \mathrm{eV}$ line. The same seems to hold for the emission due to $\mathrm{Pm}^{3+}$ observed in AlN [15].

Some doubts about the presented identification of the $1.78 \mathrm{eV}$ line are connected with its excitation process. In the case of $\mathrm{LiYF}_{4}$ : Gd the red emission was excited resonantly by UV light [3]. In our case of GaN:Gd crystals, the red emission can be excited using both above band-gap UV $(325 \mathrm{~nm})$ as well as green light $(523 \mathrm{~nm})$. This would suggest that some specific Gd-related defect levels, appearing in the band-gap of GaN, could be responsible for an efficient energy transfer to $\mathrm{Gd}^{3+}$ ions $[16,17]$. This issue needs to be further clarified.

Typical spectra measured for the GaN:Gd in the energy range close to the GaN band-gap using continuous wave excitation are presented in Fig. 3.

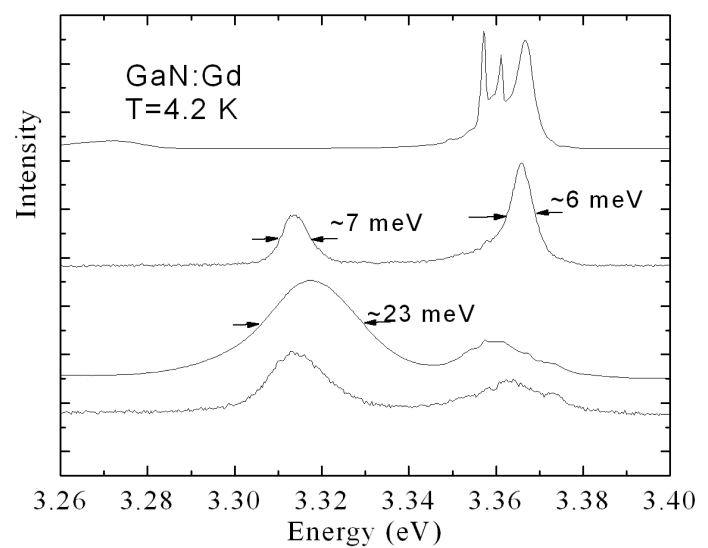

Fig. 3. Luminescence spectra measured for different spots on the GaN:Gd sample, in the UV spectral region.

It is observed that the shape and the intensity of the luminescence spectrum in this spectral region is very sensitive to the position on the sample. The decay time estimated for Gd related transitions in the UV range was found to be faster than $1 \mu \mathrm{s}$, which is close to the limit of our experimental system (see Fig. 4).

This observation remains in a strong contrast to the decay time observed for ${ }^{6} P_{7 / 2} \rightarrow{ }^{8} S_{7 / 2}$ transition in AlN, which was found to be of about $0.7 \mathrm{~ms}$ [18] and suggests that the GaN:Gd related emission lines observed in the UV region could hardly be correlated with internal transitions within $\mathrm{Gd}^{3+}\left(4 f^{7}\right)$. It seems possible that Gd ions form some complexes, for example with oxygen, which give rise to excitonic-like transitions below the band-gap of GaN:Gd, and such transitions could be responsible for the discussed UV luminescence. This conclusion is also consistent with the micro-PL mapping, which showed that the $1.78 \mathrm{eV}$ emission 


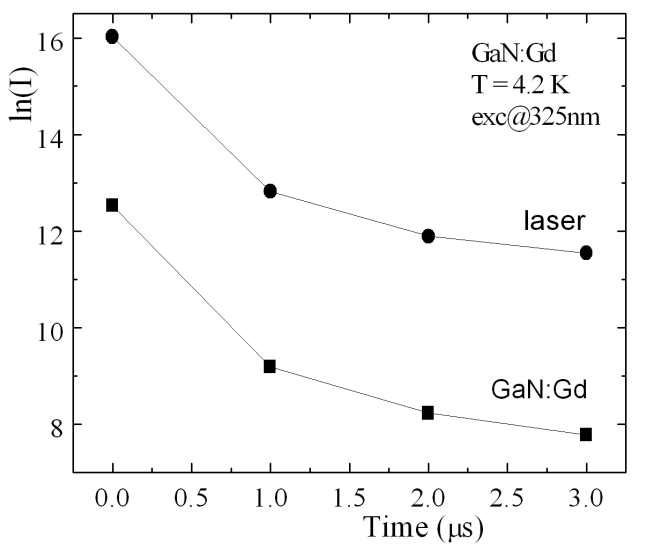

Fig. 4. A comparison of the decay of the exciting UV laser light (upper curve) with a typical transient of the Gd-related luminescence measured in the near-band-gap region (lower curve).

was not spatially correlated with presence of the Gd related lines detected in the UV region.

\section{Conclusions}

Time-resolved luminescence experiments performed on bulk GaN crystals doped with Gd revealed very long decay time of the Gd-related transitions observed in the red part of the spectrum. This supports the interpretation of the $1.78 \mathrm{eV}$ line as due to internal $\mathrm{Gd}^{3+}\left(4 f^{7}\right)$, nevertheless firm identification of this transition needs to be still confirmed. Gadolinium related emission structures observed in the GaN band-gap region seem to be related to some complexes involving Gd.

\section{Acknowledgments}

This work was partially supported by EU research framework program MTKD-CT-2005-029671 and European Commission in the 6th framework program "Transnational Access — Specific Support Action", contract No. RITA-CT-2003505474.

\section{References}

[1] S. Dhar, O. Brandt, M. Ramsteiner, V.F. Sapega, K.H. Ploog, Phys. Rev. Lett. 94, 037205 (2005).

[2] Z. Lipińska, M. Pawłowski, H. Żołnierowicz, A. Wysmołek, M. Palczewska, M. Kamińska, A. Twardowski, M. Boćkowski, I. Grzegory, Acta Phys. Pol. A 110, 243 (2006).

[3] R.T. Wegh, H. Donker, A. Meijerink, R.J. Lamminmäki, J. Hölsä, Phys. Rev. B 56, 13841 (1997). 
[4] J.B. Gruber, B. Zandi, H.J. Lozykowski, W.M. Jadwisienczak, J. Appl. Phys. 91, 2929 (2002).

[5] H.J. Lozykowski, W.M. Jadwisienczak, Appl. Phys. Lett. 76, 861 (2000).

[6] H.J. Lozykowski, W.M. Jadwisienczak, I. Brown, Appl. Phys. Lett. 74, 1129 (1999).

[7] E.E. Nyein, U. Hömmerich, J. Heikenfeld, D.S. Lee, A.J. Steckl, J.M. Zavada, Appl. Phys. Lett. 82, 1655 (2003).

[8] T. Monteiro, J. Soares, M.R. Correia, E. Alves, J. Appl. Phys. 89, 6183 (2001).

[9] K. Wang, R.W. Martin, E. Nogales, P.R. Edwards, K.P. O’Donnel, K. Lorenz, E. Alves, I.M. Watson, Appl. Phys. Lett. 89, 131912 (2006).

[10] J.H. Kim, N. Shepherd, M. Davidson, P.H. Holloway, Appl. Phys. Lett. 83, 641 (2003).

[11] K. Lorentz, U. Wahl, E. Alves, E. Nogales, S. Dalmasso, R.W. Martin, K.P. O'Donnell, M. Wojdak, A. Braud, T. Monteiro, T. Wojtowicz, P. Ruterana, S. Ruffenach, O. Briot, Opt. Mater. 28, 750 (2006).

[12] J.H. Kim, P.H. Holloway, Appl. Phys. Lett. 85, 1689 (2004).

[13] E.D. Readinger, G.D. Metcalfe, H. Shen, M. Wraback, Appl. Phys. Lett. 92, 061108 (2008).

[14] H.J. Lozykowski, W.M. Jadwisienczak, I. Brown, J. Appl. Phys. 88, 210 (2000).

[15] U. Vetter, J.B. Gruber, A.S. Nijjar, B. Zandi, G. Öhl, U. Wahl, B. de Vries, H. Hofsäss, M. Dietrich, and the ISOLDE Collaboration, Phys. Rev. B 74, 205201 (2006).

[16] J.-S. Filhol, R. Jones, M.J. Shaw, P.R. Briddon, Appl. Phys. Lett. 84, 2841 (2004).

[17] Y.E. Romanyuk, L.D. Kranz, S.R. Leone, J. Appl. Phys. 103, 073104 (2008).

[18] U. Vetter, J. Zenneck, H. Hofsäss, Appl. Phys. Lett. 83, 2145 (2003). 